\title{
Trend of HIV and tuberculosis co-epidemics in different regions of World Health Organization during 2003-2017
}

\author{
Ahmad Mehri ${ }^{1}$, Yousef Alimohamadi², Mohsen Mohammadi ${ }^{1}$, Mojtaba Sepandi ${ }^{3}$, Farzad Khodamoradi ${ }^{4}$, \\ Firooz Eesmaeilzadeh ${ }^{5}$ \\ ${ }^{1}$ Department of Epidemiology and Biostatistics, School of Public Health, Tehran University of Medical Sciences, Tehran, Iran \\ ${ }^{2}$ Pars Advanced and Minimally Invasive Medical Manners Research Center, Pars Hospital, Iran University of Medical Sciences, Tehran, Iran \\ ${ }^{3}$ Health Research Center, Lifestyle Institute, Baqiyatallah University of Medical Sciences, Tehran, Iran \\ ${ }^{4}$ Department of Community Medicine, School of Medicine, Dezful University of Medical Sciences, Dezful, Iran \\ ${ }^{5}$ Department of Public Health, School of Public Health, Maragheh University of Medical Sciences, Maragheh, Iran
}

\begin{abstract}
Introduction: The co-infection of human immunodeficiency virus (HIV)/tuberculosis (TB) is one of the main public health problems globally. Due to the importance of this problem, the aim of the current study was to determine the trend of TB patients with known HIV status and TB patients who are HIV-positive in different regions of World Health Organization (WHO) from 2003 to 2017.

Material and methods: In the current ecological study, the needed information about the HIV/TB cases were extracted from the WHO website. To assess the trend of changes over the time of understudy variables, the Spearman correlation coefficient and figures were used. The statistical significance level for this study was considered as $p=0.05$. All analysis was performed by Excel 2010 and SPSS version 22 .

Results: The percentage of TB patients with known HIV status during 2003-2017 had an increasing trend in all parts of the WHO regions. The HIV/TB co-infection showed a decreasing tendency for all regions, except Europe. Furthermore, the trend of HIV/TB co-infection in Europe was increasing during the studied period.

Conclusions: Given that the diseases are global, not only countries should expand education and investing in HIV and tuberculosis control, but also international community organizations should attempt to identify and treat the infected patients in underdeveloped and developing countries.
\end{abstract}

HIV AIDS Rev 2020; 19, 3: 167-171

DOI: https://doi.org/10.5114/hivar.2020.99688

Key words: co-infection, HIV/TB, WHO regions.

\section{Introduction}

Globally, tuberculosis (TB) and human immunodeficiency virus (HIV) co-epidemic are one of the main problems of public health [1]. TB infection is one of the major causes of mortality among HIV patients [2,3]. Due to weak- ening of the body immune system in HIV infection [4], opportunistic infections, such as TB, can threaten the life of patient and even be the cause of death [5]. Among infectious diseases, both TB and HIV are considered as the greatest public health problems $[6,7]$. The emergence of HIV pandemic has led to a significant increase in the number of peo-
Address for correspondence: Dr. Firooz Eesmaeilzadeh, Department of Public Health, School of Public Health, Maragheh University of Medical Sciences, Maragheh, Iran, e-mail: firooz.esmaeilzadeh@gmail.com
Article history:

Received: 23.06.2019

Received in revised form: 08.09.2019

Accepted: 09.10.2019

Available online: 30.07 .2020
International Journal of HIV-Related Problems

HIV \& AIDS R e vi e w 
ple with TB worldwide. Generally, one of the most important causes of mortality and morbidity in HIV patients is TB [8]. According to the World Health Organization (WHO), HIV and TB are the first and the second causes of death from a single infection agent in the world [9]. The interaction between HIV and TB is overly complicated. HIV diminishes the immune system, therefore predisposing to TB infection. Additionally, HIV increases the likelihood of reactivation, re-infection, and the latent infection of TB leads to active disease and challenges in TB treatment [10]. It was estimated that about 10.4 million TB cases occurred in 2016, with 1.3 million deaths among HIV-negative people and 374,000 extra deaths among HIV-positives [11]. In a population, lifetime risk for development of active TB for infected people with TB is $5-15 \%$. HIV-infected people are 26-31 times more likely to be affected by active TB $[10,11]$. Moreover, TB and HIV are strongly associated with malnutrition, unemployment, alcoholism, drug abuse, poverty, and homelessness. The direct and indirect costs of these two diseases are exceptionally large, involving more than $30 \%$ of the annual household income in developing countries, which is a major impact on the economy of developing world [8].

Due to many problems associated with the HIV/TB co-infection in different regions of the world, and that it is very important to know the effects of the measures taken over time in order to control and prevent these diseases, the aim of the current study was to determine the trend of TB patients with known HIV status and TB patients who are HIV-positive in different parts of WHO from 2003 to 2017.

\section{Material and methods}

In the current ecological study, the data concerning the percentage of all registered TB patients with documented
HIV status, and HIV-positive and TB patients with known HIV status was extracted from the World Health Organization (WHO) website: http://apps.who.int/gho/data/view. main.TBHIVWHOREG?lang=en. The WHO in the Global Health Observatory (GHO) is collecting health data from 194 member countries. This information is extracted from countries' health care systems every few years and is published by the WHO. Out of this data, HIV status records, HIV-positive, and TB patients with known HIV status are included in our study. Inclusion criteria consist of registry information related to persons of 15 years of age or older and reported in the countries' health system.

\section{Measurements}

The percentage of all registered TB patients with documented HIV status is defined as the percentage of registered TB patients with documented HIV-positive status in TB register, expressed as a proportion of the total number of all registered TB patients with documented HIV status, over the reporting period. Also, HIV-positive and TB patients with known HIV status are defined as the percentage of TB patients, who had an HIV test result recorded in the TB register. Both indexes are preferred from surveillance systems and method of estimation, with facilities TB registers and quarterly case-finding reports.

\section{Statistical analysis}

The descriptive methods, such as tables and figures, were used to show the feature of understudy data. Additionally, to assess the trend of changes over time of understudy variables, the Spearman correlation coefficient was used. The statistical significance level for this study was consid-

Table 1. Percentage of tuberculosis patients with known HIV status in different regions during 2003-2017

\begin{tabular}{|c|c|c|c|c|c|c|c|}
\hline Year & Africa & Americas & South-East Asia & Europe & Eastern Mediterranean & Western Pacific & Global (WHO) \\
\hline 2003 & 7.8 & 46 & 4.2 & 79 & 8.3 & 14 & 18 \\
\hline 2004 & 7.6 & 41 & 25 & 48 & 9.2 & 13 & 18 \\
\hline 2005 & 14 & 39 & 2.1 & 58 & 4.8 & 20 & 15 \\
\hline 2006 & 26 & 35 & 4.7 & 60 & 5.5 & 2.3 & 14 \\
\hline 2007 & 39 & 44 & 7.1 & 82 & 7 & 8.8 & 23 \\
\hline 2008 & 50 & 45 & 3.9 & 86 & 7 & 11 & 23 \\
\hline 2009 & 57 & 49 & 14 & 80 & 11 & 12 & 29 \\
\hline 2010 & 61 & 54 & 25 & 58 & 10 & 20 & 35 \\
\hline 2011 & 70 & 56 & 34 & 60 & 11 & 26 & 42 \\
\hline 2012 & 75 & 62 & 41 & 64 & 13 & 34 & 47 \\
\hline 2013 & 79 & 72 & 45 & 66 & 11 & 36 & 51 \\
\hline 2014 & 81 & 76 & 47 & 68 & 15 & 41 & 53 \\
\hline 2015 & 83 & 81 & 54 & 75 & 17 & 44 & 57 \\
\hline 2016 & 84 & 81 & 58 & 86 & 16 & 42 & 58 \\
\hline 2017 & 86 & 81 & 55 & 91 & 21 & 51 & 60 \\
\hline
\end{tabular}




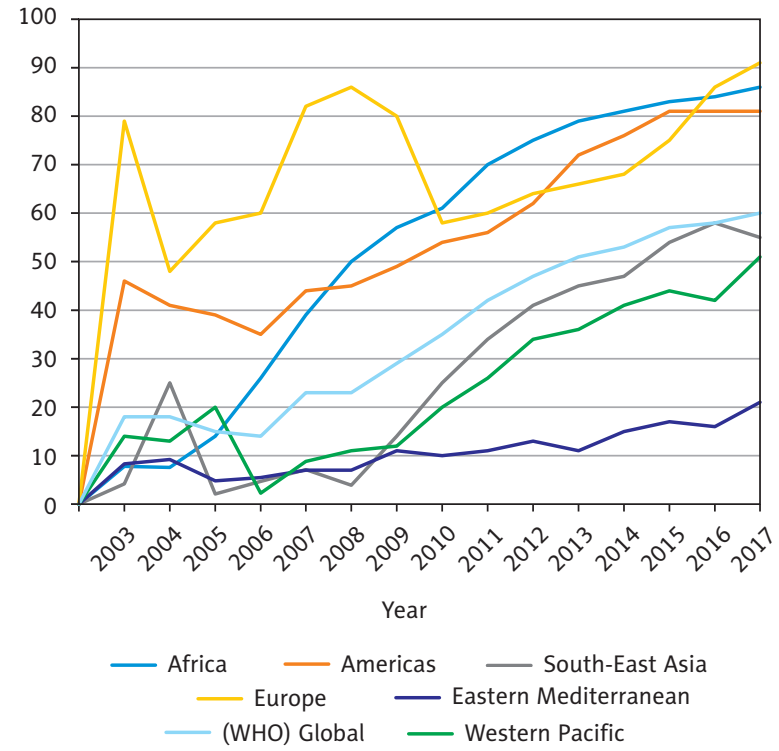

Figure 1. The trend of tuberculosis patients with known HIV status in different regions during 2003-2017

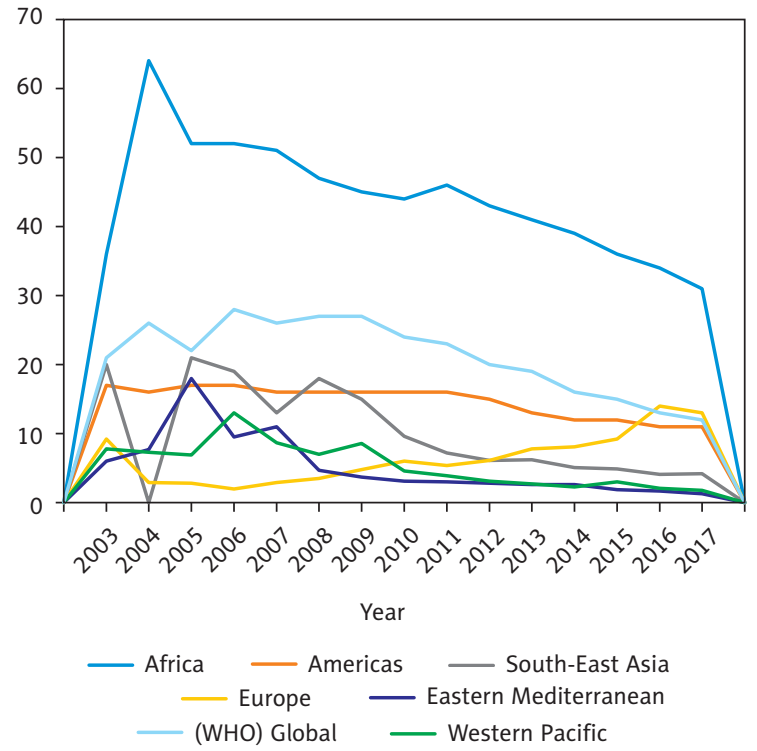

Figure 2. The trend of all registered tuberculosis patients who were HIV-positive in different regions during 2003-2017

Table 2. Percentage of all registered tuberculosis patients who were HIV-positive in different regions during 2003-2017

\begin{tabular}{l|c|c|c|c|c|c|c}
\hline Year & Africa & Americas & South-East Asia & Europe & Eastern Mediterranean & Western Pacific & Global (WHO) \\
\hline 2003 & 36 & 17 & 20 & 9.2 & 6 & 7.8 & 21 \\
\hline 2004 & 64 & 16 & & 2.9 & 7.7 & 7.3 & 26 \\
\hline 2005 & 52 & 17 & 21 & 2.8 & 18 & 6.9 & 22 \\
\hline 2006 & 52 & 17 & 19 & 2 & 9.5 & 13 & 28 \\
\hline 2007 & 51 & 16 & 13 & 2.9 & 11 & 8.7 & 26 \\
\hline 2008 & 47 & 16 & 18 & 3.5 & 4.7 & 3.7 & 27 \\
\hline 2009 & 45 & 16 & 15 & 4.8 & 6.6 & 4.6 & 27 \\
\hline 2010 & 44 & 16 & 9.6 & 6 & 3.1 & 3.9 & 23 \\
\hline 2011 & 46 & 16 & 7.2 & 5.4 & 3.1 & 20 \\
\hline 2012 & 43 & 15 & 6.1 & 6.1 & 2.8 & 2.7 & 19 \\
\hline 2013 & 41 & 13 & 6.2 & 7.8 & 2.6 & 2.3 & 16 \\
\hline 2014 & 39 & 12 & 5.1 & 8.1 & 2.6 & 3 & 15 \\
\hline 2015 & 36 & 12 & 4.9 & 9.2 & 1.9 & 2.1 & 13 \\
\hline 2016 & 34 & 11 & 4.1 & 14 & 1.7 & 1.8 & 12 \\
\hline 2017 & 31 & 11 & 4.2 & 13 & 1.3 & & 7 \\
\hline
\end{tabular}

ered as $p=0.05$. All analysis was performed by Excel 2010 and SPSS version 22.

\section{Results}

\section{Tuberculosis patients with known HIV status}

Although the trend of percentage of TB patients with known HIV status had increased during 2003-2017 in all WHO regions, the highest level for this index was in the African, European, and Americans regions during this period.
Also, the Eastern Mediterranean, Western Pacific, and SouthEast Asian regions presented the lowest levels among all regions (Table 1 and Figure 1). According to the results, the European region had the highest percentage of $\mathrm{TB}$ patients with known HIV status until 2009 (80\%), but after that, the African region had the highest percentage increase until 2015 $(86 \%)$. Also, the European region had the highest percentage of TB patients with known HIV status among other regions during 2015-2017.

In the Americans region, the percentage of TB patients with known HIV status increased from $46 \%$ to $81 \%$ during 
Table 3. The trend analysis of tuberculosis patients who were HIV-positive over time in different regions during 2003-2017

\begin{tabular}{l|c|c}
\hline Region & Correlation coefficient & $p$-value \\
\hline Africa & -0.72 & 0.003 \\
\hline Americas & -0.91 & $<0.001$ \\
\hline South-East Asia & -0.94 & $<0.001$ \\
\hline Europe & 0.74 & 0.002 \\
\hline Eastern Mediterranean & -0.72 & 0.002 \\
\hline Western Pacific & -0.82 & $<0.001$ \\
\hline Global (WHO) & -0.79 & $<0.001$ \\
\hline
\end{tabular}

the studied period. The lowest growth of percentage of TB patients with known HIV status was in the Eastern Mediterranean region, which increased from $8.3 \%$ to $21 \%$ during 2003 to 2017. Globally, the percentage of TB patients with known HIV status presented an increasing trend from 18\% to $60 \%$ during 2003 to 2017.

\section{HIV/TB co-infection in different regions}

In assessing the trend of HIV/TB co-infection in different regions, all regions showed decreasing trend, except for Europe. On average, the HIV/TB co-infection trend in Europe had increased during 2003-2017 (Table 2 and Figure 2).

According to the results, the trend of all registered TB patients who were HIV-positive had increased between 2005 and 2006, but between 2006 and 2017, the trend had declined in all areas. Although this index had declined in all regions, the highest percentage was noted in the African and Americans regions during 2003 to 2017. Moreover, the highest percentage of this index was found in the African region in 2017.

In case of trend analysis over time, the correlation coefficient $(r)$ of reported TB patients who were HIV-positive over time for the African, Americans, South-East Asian, Eastern Mediterranean, and Western Pacific regions were $-0.72,-0.91,-0.94,-0.72$, and -0.82 , respectively. It means that the number of reported cases over time had a decreasing trend. Also, the number for the European region was 0.74 , which means that the number of reported cases in this region had an increasing trend during the last years. All mentioned correlation coefficients were statistically significant $(p<0.05$; Table 3$)$.

\section{Discussion and conclusion}

Tuberculosis and HIV are diseases that enhance each other and the adverse effect on health increases when they co-occur. That is to say, the most common cause of death in patients with HIV is pulmonary tuberculosis, and HIV infection is one of the main causes of conversion of latent tuberculosis to active tuberculosis in patients. HIV screening in patients with tuberculosis is of particular importance, because preventive TB therapy with anti-TB drugs and antiretroviral therapy can significantly reduce the probability of tuberculosis activation in HIV patients up to $32 \%$ and $92 \%$, respectively $[12,13]$.

During the study, it was found that the percentage of tuberculosis in patients screened for HIV presented an increasing trend in all regions, and the percentage of patients simultaneously suffering from both diseases had substantially decreased. This indicates countries' attention to the danger of co-occurrence of these diseases. Given the large number of the patients with both diseases in 2017, especially in regions such as Africa, America, and Europe, such efforts must be continued even more intensely. In 2017, the highest percentages of tuberculosis in patients examined for HIV infection were found in Europe and Africa. This high prevalence of HIV in Africa requires to examine HIV-positive patients for tuberculosis. However, the other and more important reason for such a high rate in Africa might be the identification of a small number of tuberculosis patients who have an access to healthcare services, and it is likely that a large number of TB patients has not been identified in this area. According to studies, Africa has the highest rate of HIV/TB co-occurrence. According to the statistics on high rates of malnutrition, poverty, unemployment, and lack of adequate access to healthcare services in the region and their relationship with TB and HIV [14], the high prevalence of these two diseases and the their subsequent co-occurrence in Africa are not unexpected. Apart from Africa, the European region presented the highest rate of HIV/TB simultaneous infection in 2017, the reason for which could be the high prevalence of drug abuse [15] and migration, especially from sub-Saharan Africa to European countries $[15,16]$. In addition, simultaneous infection was more prevalent among European men due to having sex with men and drug injection [15].

Given that HIV and TB diseases are occurring globally, not only countries should expand education and investing in HIV and tuberculosis control, but also international community organizations should attempt to identify and treat the infected patients in underdeveloped and developing countries.

\section{Acknowledgment}

I would also like to declare that I did not receive any funding for the preparation of this article. Finally, I can affirm that I have no conflict of interest that is directly or indirectly relevant to the content of this article.

\section{Conflict of interest}

The authors declare no conflict of interest with respect to the research, authorship, and/or publication of this article.

\section{References}

1. Gao L, Zhou F, Li X, Jin Q. HIV/TB co-infection in mainland China: a meta-analysis. PLoS One 2010; 5: e10736.

2. Manosuthi W, Chottanapand S, Thongyen S, Chaovavanich A, Sungkanuparph S. Survival rate and risk factors of mortality among 
HIV/tuberculosis-coinfected patients with and without antiretroviral therapy. J Acquir Immune Defic Syndr 2006; 43: 42-46.

3. Tabatabaee H, Afsar-Kazerooni P, Alimohamadi Y, et al. Intensified or routine case finding for pulmonary tuberculosis among HIV-infected patients: which is better? J Health Sci Surveill Syst 2015; 3: 13-19.

4. Alimohamadi Y, Tabatabaee H, Afsarkazerooni P, Vahedi S, Enaami M, Teimourizad A. Epidemiologic characteristics of HIV-positive patients referring to behavioral diseases consultation center in Shiraz, Iran. Med J Islam Repub Iran 2014; $28: 147$.

5. Toossi Z, Mayanja-Kizza H, Hirsch C, et al. Impact of tuberculosis (TB) on HIV-1 activity in dually infected patients. Clin Exp Immunol 2001; 123: 233-238.

6. Haseli N, Esmaeelzadeh F, Ghahramani F, Alimohamadi Y, Hayati R, Mahboubi M. Health-related quality of life and its related factors in HIV+ patients referred to Shiraz Behavioral Counseling Center, Iran in 2012. Med J Islam Repub Iran 2014; $28: 13$.

7. Trinh Q, Nguyen H, Nguyen V, Nguyen T, Sintchenko V, Marais B. Tuberculosis and HIV co-infection - focus on the Asia-Pacific region. Int J Infect Dis 2015; 32: 170-178.

8. Sharma S, Mohan A, Kadhiravan T. HIV-TB co-infection: epidemiology, diagnosis \& management. Indian J Med Res 2005; 121 : 550-567.

9. Silva CJ, Torres DF. A TB-HIV/AIDS coinfection model and optimal control treatment. arXiv: 1501.03322.

10. Datiko DG, Yassin MA, Chekol LT, Kabeto LE, Lindtjørn B. The rate of TB-HIV co-infection depends on the prevalence of HIV infection in a community. BMC Public Health 2008; 8: 266.

11. Gelaw YA, Williams G, Magalhães RJS, Gilks CF, Assefa Y. HIV prevalence among tuberculosis patients in sub-Saharan Africa: a systematic review and meta-analysis. AIDS Behav 2019; 23: 15611575.

12. Gao J, Zheng P, Fu H. Prevalence of TB/HIV co-infection in countries except China: a systematic review and meta-analysis. PLoS One 2013; 8: e64915.

13. Manjareeka M, Nanda S. Prevalence of HIV infection among tuberculosis patients in Eastern India. J Infect Public Health 2013; 6: 358-362.

14. Pennap G, Makpa S, Ogbu S. Sero-prevalence of HIV infection among tuberculosis patients in a rural tuberculosis referral clinic in northern Nigeria. Pan Afr Med J 2010; 5: 22.

15. Pimpin L, Drumright LN, Kruijshaar ME, et al. Tuberculosis and HIV co-infection in European Union and European Economic Area countries. Eur Respir J 2011; 38: 1382-1392.

16. Tavares AM, Fronteira I, Couto I, et al. HIV and tuberculosis coinfection among migrants in Europe: a systematic review on the prevalence, incidence and mortality. PLoS One 2017; 12: e0185526. 